\title{
Comparison with Low-Velocity Impact and Quasi-static Indentation Testing of Foam Core Sandwich Composites
}

\author{
Yan Li, An Xuefeng, and Yi Xiaosu
}

\begin{abstract}
The need for quasi-static indentation test method for modeling low-velocity foreign object impact events would prove to be very beneficial to researchers. In order to examine whether it is feasible, series of quasi-static indentation and low-velocity impact tests were carried out and compared. An analysis of the relationships between impact energy (or quasi-static indentation force) and damage area, dent depth indicates clearly that dent depth was selected as the damage parameter to set up damage relationship between the two tests. The knee points of dent depth appearing in the two tests curves were very close. The variation tendency of the dent depth, the process curves and the cross sectional damage views of the two tests were in similarity. Results show that no distinct differences could be seen between low-velocity impact and quasi-static indentation testing, indicating that quasi-static indentation testing can be used to represent low-velocity impact testing.
\end{abstract}

Index Term-Sandwich composites; low-velocity impact; quasi-static indentation; dent depth.

\section{INTRODUCTION}

Composite sandwich panels are widely used in aerospace, marine, automotive and recreational industries, due to the high specific strength and stiffness, corrosion-resistance, tailorability and stability [1]. Sandwich composites structures consist of two thin fiber reinforced composite face sheets, which are bonded to a lightweight foam core. The function of the face sheets is to carry bending and inplane forces, while the role of the core is to keep the face sheets together and carry transverse shear loads. One of the main concerns in the application of sandwich composite structures is that their load carrying ability may be significantly reduced by the presence of a local damage (delamination) between the core and the face. A frequent cause of such damage is in-service incidents (runway debris, careless handling, etc.) or interactions with attached structures (pillars, bends, etc) [2-4] It should be noted that such damages of sandwich composites usually cannot be observed outside. However, they may cause serious reduction in load-bearing capacity. Thus, it is of great practical importance to develop numerical models capable of characterizing the response of sandwich structures subjected to local loading.

The need for quasi-static indentation test method for modeling low-velocity foreign object impact events to sandwich composites would prove to be very beneficial to researchers since much more data can be obtained from

Manuscript received September 10, 2011; revised December 20, 2011.

This work was supported in part by the Weapon Equipment Pre-Research Foundation of China under grant No. 9140C4405041003.

Y. Li, A. Xuefeng, and Y. Xiaosu are with Science and Technology on Advanced Composites Laboratory, Beijing Institute of Aeronautical Materials (e-mail: yanli0827@163.com). quasi-static indentation testing than from low-velocity impact testing. Several studies [5-10] show a similarity between quasi-static indentation and drop weight impact testing. While other studies[11-13] have shown a limit to the applicability of using quasi-static indentation to represent low velocity impact. It must be noted that there are many variables involved in these tests such as boundary conditions, specimen size, specimen thickness, stacking sequence, impactor size, impactor shape and type of fiber/resin system. Such studies on the comparison of quasi-static indentation to low velocity impact testing are with respect to composite laminates. But the relevant researches about sandwich composite panels are few relatively. An experimental investigation by Ferri and Sankar [14] has revealed that the contact force-indentation relations for a quasi-static test and a low-velocity impact event about sandwich composites are virtually equivalent. Lindholm C-J studied impact and indentation behavior of sandwich panels [15]. Such studies indicate that it is limited to reflect the relationship between low-velocity impact testing and quasi-static indentation testing.

The method of assessing damage resistance using low-velocity impact tests is time-consuming and expensive, and the test results have large dispersibility. By simulating an impact event using a quasi-static indentation test, damage initiation and propagation can be more easily detected, and deflection can be directly measured with great accuracy and maximum transverse force can be better controlled. Low-velocity impact test of composites accepted ASTM D7136-05 standard [16], and quasi-static test of composites adopted ASTM D6264-98 standard [17]. In this study, it is the focus of the work to examine if drop weight impact tests and quasi-static indentation tests give the same tests conditions (size, shape, and location of damage for a given maximum transverse load) according to the same ASTM standard. It is of significance for learning and simulating the damage of foam core sandwich composite panels.

\section{EXPERIMENTAL STUDY}

The core of sandwich panels was constituted of Rohacell 71WF-HT closed cell polymethacrylimide (PMI) foam made by Röhm foam $\mathrm{GmbH}$. The nominal density of the foam was $75 \mathrm{~kg} / \mathrm{cm}^{3}$. One of the advantages of the Rohacell foam was its relatively high temperature resistance. The investigated specimens consisted of relatively thin $(4 \mathrm{~mm})$ foam core. The core was sandwiched between two face sheet carbon fiber laminates with thickness of $1.52 \mathrm{~mm}$. The face sheets were produced from T700 unidirectional textile with an areal density of $192 \mathrm{~g} / \mathrm{m}^{2}$ and bismaleimide (BMI) resin. Quasi-isotropic and symmetric lay-up $[45 / 0 /-45 / 90]_{s}$ was 
chosen. Sandwich composite panels were manufactured by resin transfer molding (RTM) process. Rectangular specimens cut from the panels with in-plane size of 150 $\mathrm{mm} \times 100 \mathrm{~mm}$ were used.

The two categories of tests are depended on the test conditions. The sample size, supported mode and impactor/indenter size of the two test standards are inconsistent. In order to compare with the two tests, drop weight test standard was used as the basement. So the sample size, supported mode and impactor/indenter size were according to ASTM D 7136-05. The sample size was $150 \mathrm{~mm} \times 100 \mathrm{~mm}$, and the specimens were clamped on all four edges of $125 \mathrm{~mm} \times 75 \mathrm{~mm}$ rectangular opening size on center. The steel impactor/indenter used for the tests has a hemispherical strike tip with a $16 \mathrm{~mm}$ diameter.

The impact testing was performed using a drop-weight impact tester (INSTRON 9250HV). The drop-weight testing machine consists of a drop tower equipped with an impactor, which has high bandwidth digital signal processing (DSP) electronics, Impulse TM control and data acquisition software. And the quasi-static indentation testing was performed using a electronic tensile tester (INSTRON 5569) with loading speed $1.25 \mathrm{~mm} / \mathrm{s}$.

In this test, damage area and dent depth will be measured.. After the specimens were impacted or subjected to quasi-static indentation testing, evaluation of the impact damage inflicted to the specimens is performed by means of ultrasonic C-scan. The samples were set aside for at least 24 hours so that the resulting dent depth would have enough time to relax to its equilibrium state. The dent depth was measured by vernier calipers.

\section{RESUlts AND DisCUSSION}

\section{A. Analysis of Damage Parameters}

Once the impact testing was completed, the maximum impact force obtained for each of the different subgroups was used as the independent variable for the quasi-static indentation testing. Fig. 1 shows the damage area obtained from $\mathrm{C}$-scan as a function of impact energy (quasi-static indentation force) for the sandwich composite panels. As shown in Fig.1 (a), the data dispersibility of damage area versus impact energy and is large. Also, regularity cannot be found, and did not reflect the damage of sandwich composites directly. As indicated in Fig.1 (b), with increasing of the quasi-static indentation force, damage area increased gradually. Due to dispersibility of the data, it could not be found whether there was little resemblance between the change trend of the impact energy (quasi-static indentation force) versus damage area curves. So damage area is not suitable as damage parameter to establish the equivalence of low-velocity impact testing and quasi-static indentation testing.

The relationship between impact energy (quasi-static indentation force) and dent depth was presented in Fig.2. The curves of impact energy versus dent depth, quasi-static indentation force versus dent depth had good regularity and corresponding relationship. Dent depth could reflect the changes of the damage resistance of sandwich composites.
As shown in Fig.2 (a), with increasing of the impact energy, the dent depth increased gradually. The dent depth was increased from $0.18 \mathrm{~mm}$ to $0.27 \mathrm{~mm}$ only when the impact energy rose from $5 \mathrm{~J}$ to $15 \mathrm{~J}$. When the impact energy reached $20 \mathrm{~J}$, knee point appeared. After that, the impact energy has a large influence on the dent depth. As the impact energy increasing from $15 \mathrm{~J}$ to $40 \mathrm{~J}$, the dent depth also grew from $0.30 \mathrm{~mm}$ to $0.55 \mathrm{~mm}$. As shown in Fig.2 (b), it is apparent that the curve of quasi-static indentation force and dent depth has similar tendency to Fig.2 (a). During the stage of lower load, the dent depth has risen up to $0.27 \mathrm{~mm}$, when the quasi-static indentation force reached $4000 \mathrm{~N}$. After the knee point, the dent depth has increased promptly. The knee points of the dent depth of the two tests were very close and the variation tendency is identical, which indicated that quasi-static indentation tests may replace low-velocity impact tests. As indicated in Fig.2 (c), damage area after low-velocity impact and quasi-static indentation testing was equal approximately when the impact energy was less than $12 \mathrm{~J}$, while dent depth had much more difference at higher impact energy. So when the impact energy was lower (about less than $12 \mathrm{~J}$ ), quasi-static indentation force was seen as approximately equal to the maximum force caused by impact process of corresponding impact energy.

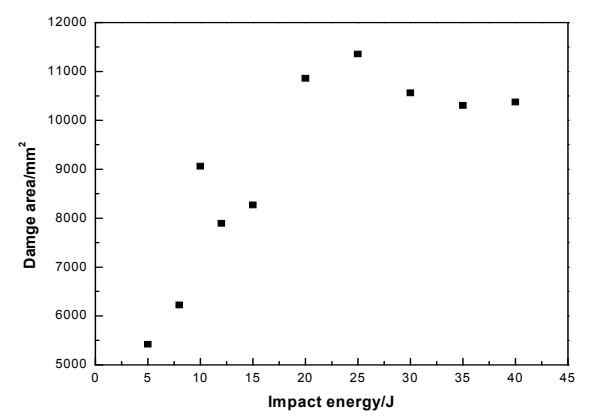

(a) Low-velocity impact testing

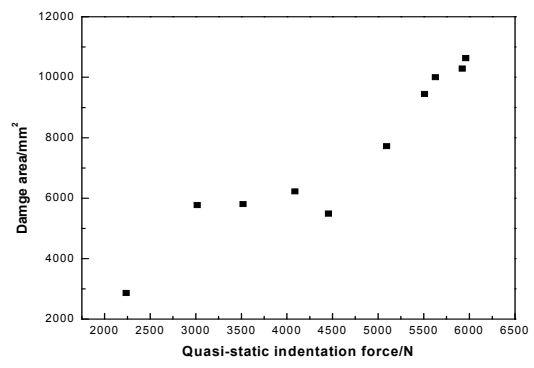

(b) Quasi-static indentation testing

Fig. 1. Relationship between impact energy (or quasi-static indentation force) and damage area.

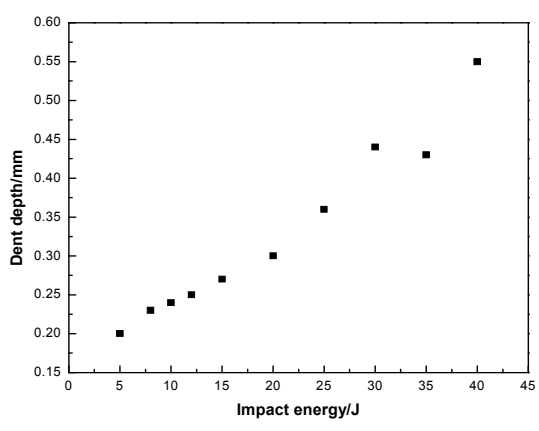

(a) Low-velocity impact testing 


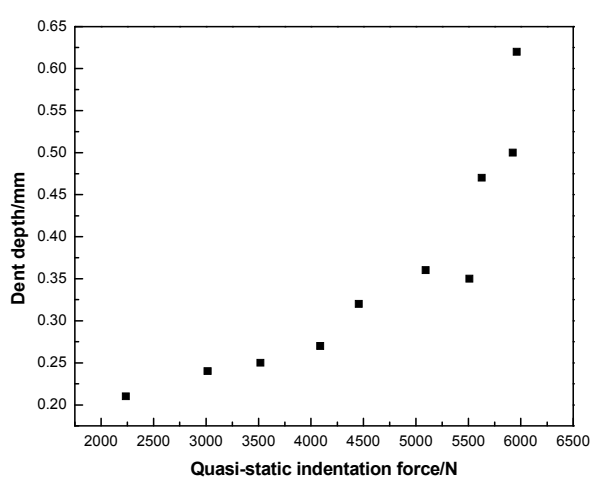

(b) Quasi-static indentation testing

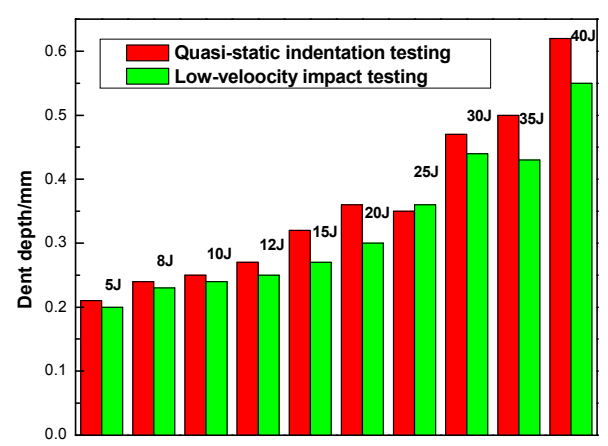

Fig. 2. Relationship between impact energy (or quasi-static indentation force) and dent depth.

As presented in Fig. 1 and Fig. 2, the testing data of impact energy versus damage area, quasi-static indentation force versus damage area has large dispersibility. However, the curves of impact energy versus dent depth, quasi-static indentation force versus dent depth had good corresponding relationship. Compared with damage area, dent depth was proper to be damage parameter to reflect the changes of damage resistance of sandwich composites. Z. Shen, et al. [18]-[21] have found dent depth is most sensitive to the capability of resistance to impact of composites, and dent depth could reflect the changes of the damage resistance (toughness) of composites obviously. At the meantime, compared with damage area and other damage parameters, the testing data of dent depth has smallest dispersibility and dent depth is a visible impact damage parameter easiest to be measured. In other words, dent depth could be as the damage parameter to establish the equivalence of impact energy and quasi-static indentation testing.

\section{B. Damage Process of Low-Velocity Impact and Quasi-Static Indentation Testing}

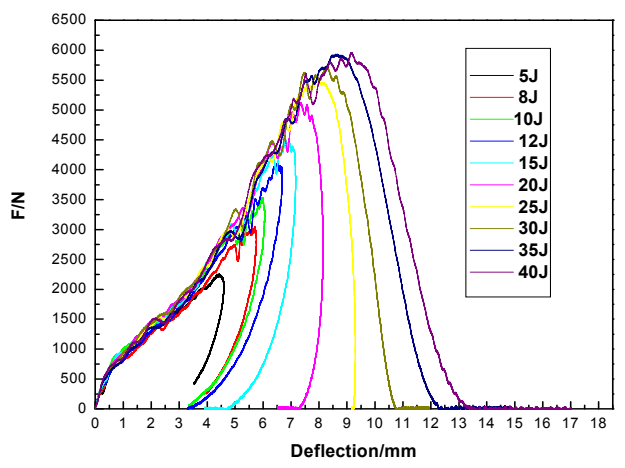

Fig. 3. Force-Deflection curves during impact.

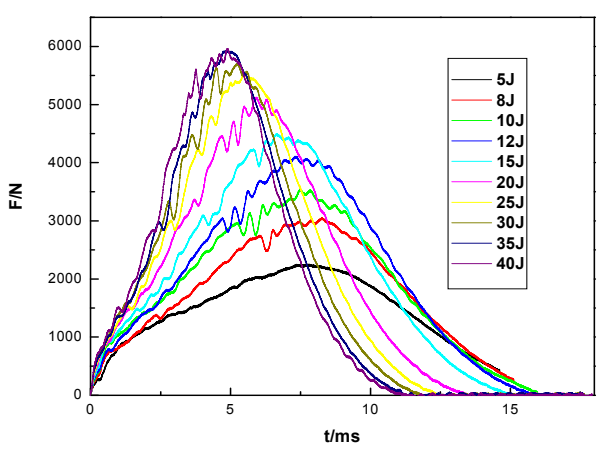

Fig. 4. Force-Time curves during impact.

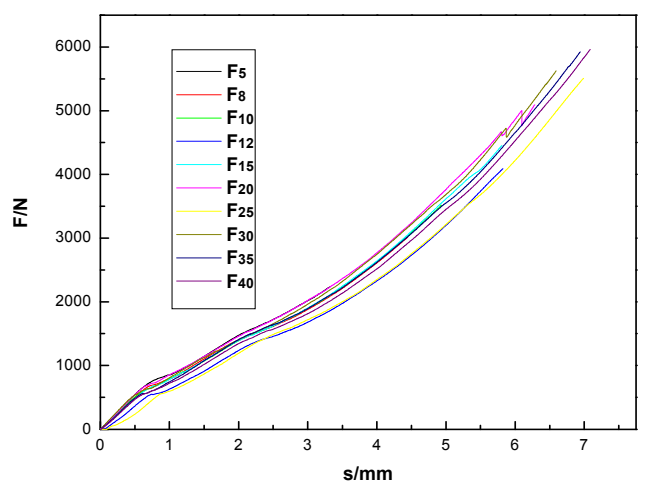

Fig. 5. Force-Displacement curves during quasi-static indentation.

As presented in Fig. 3-Fig. 5, the curves of damage process of low-velocity impact and quasi-static indentation testing had the similar variation tendency. Initially the force increased gradually in linearity with the growth of displacement. When the displacement was up to $0.5 \mathrm{~mm}$ and the force reached near $650 \mathrm{~N}$, the knee point appeared in low-velocity impact testing. While the displacement was 0.6 $\mathrm{mm}$ and the force was near $550 \mathrm{~N}$, the knee point occured in quasi-static indentation testing. The knee point appeared in the two kinds of tests was very close. Then the force grew rapidly relatively in index similarly until the maximum. The knee point onset load would increase and the time when the knee point appearing was in advance as the impact energy (quasi-static indentation force) increased. Different impact energy was selected randomly to compare with the two kinds of tests, as in Fig.6. The curves of low-velocity impact and quasi-static indentation testing were in good similarity. In general, the resemblance of the process curves of the two kinds of tests indicated the damage equivalence of sandwich composite panels subjected to low-velocity impact and quasi-static indentation tests.

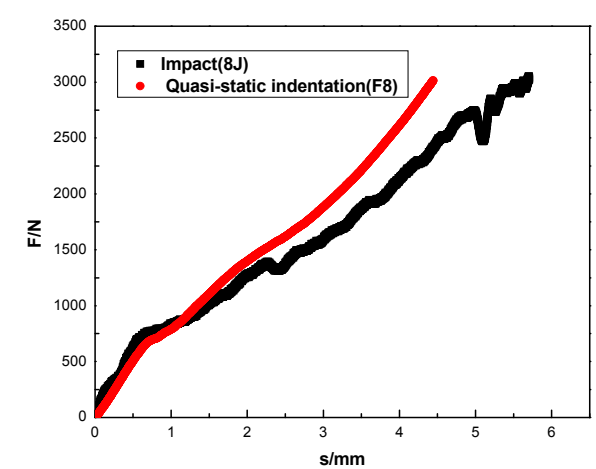

(a) $8 \mathrm{~J}$ 


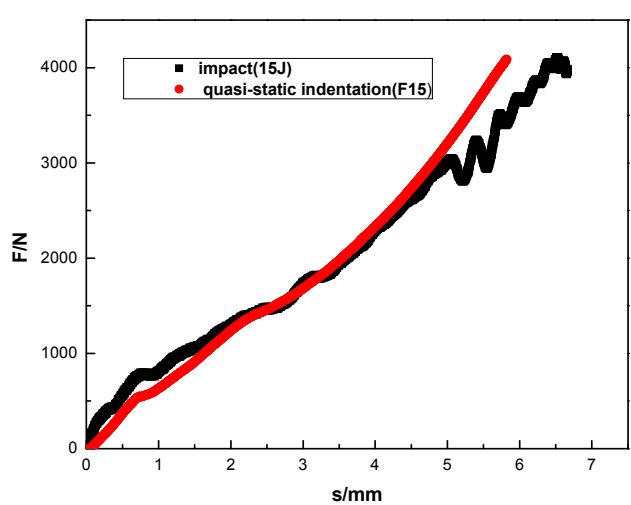

(b) $15 \mathrm{~J}$

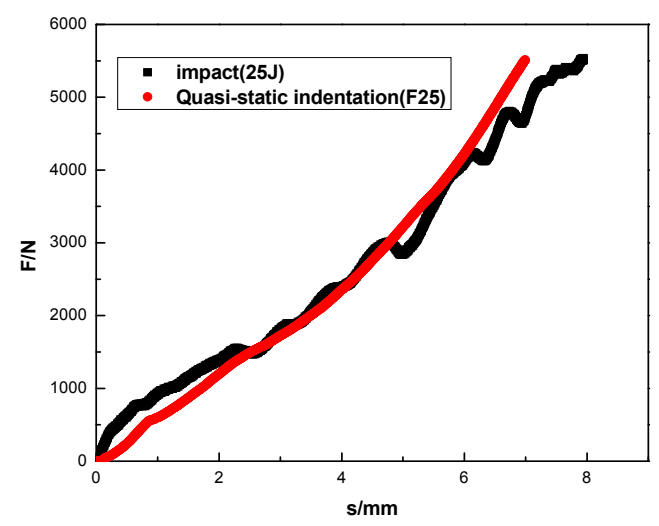

(c) $25 \mathrm{~J}$

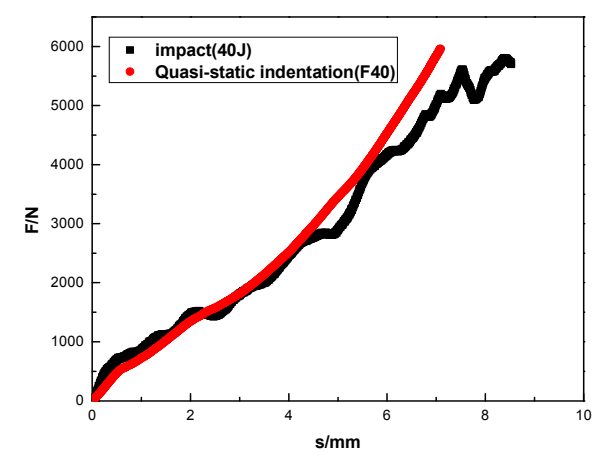

(d) $40 \mathrm{~J}$

Fig. 6. Comparison of low-velocity impact and quasi-static indentation testing process.

\section{Inspection of Specimens}

The specimens after low-velocity impact and quasi-static indentation testing were cut through the impact (or static indentation) location for visual and microscopic investigation of the sustained damage. The cross-sections reveal that delamination and fiber breakage only occur in the upper (impacted) facing. In addition, local crushing of foam core could clearly be observed. The bottom facing is left damaged. Fig. 7 displays the cross sectional views of both halves of the sectioned specimens subjected to low-velocity impact and quasi-static indentation testing. The damage process of the two kinds of tests was similar as shown in Fig.7 (a) and Fig.7 (b). Initially collapse of foam appeared, while the knee point appeared as shown in Fig. 3-Fig. 5. With increasing of the impact energy (or quasi-static indentation force), the collapse height of foam had grown gradually and the damage area was also increasing. And then foam generated crack which extended in transverse direction. Finally fiber breakage and delamination appeared on the upper facing when the load was up to the maximum force. From the cross-sections, the damage behavior of the two kinds of tests at low impact energy was similar, a little different at high impact energy, so quasi-static indentation force was seen as approximately equal to the maximum force caused by impact process of corresponding impact energy during the stage of low impact energy.
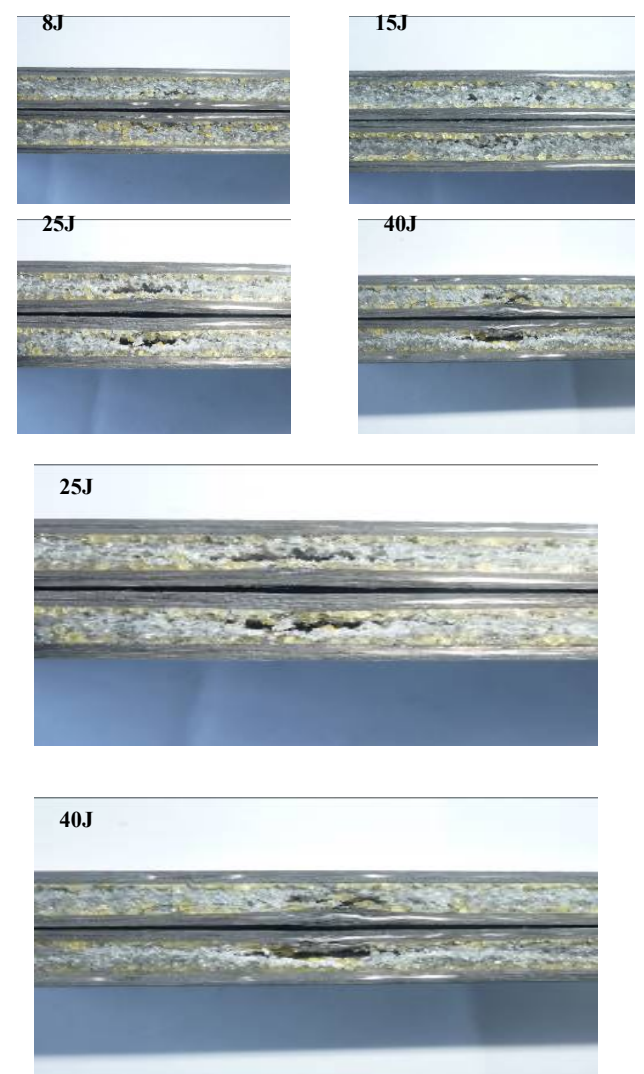

(a) Low-velocity impact testing

F8
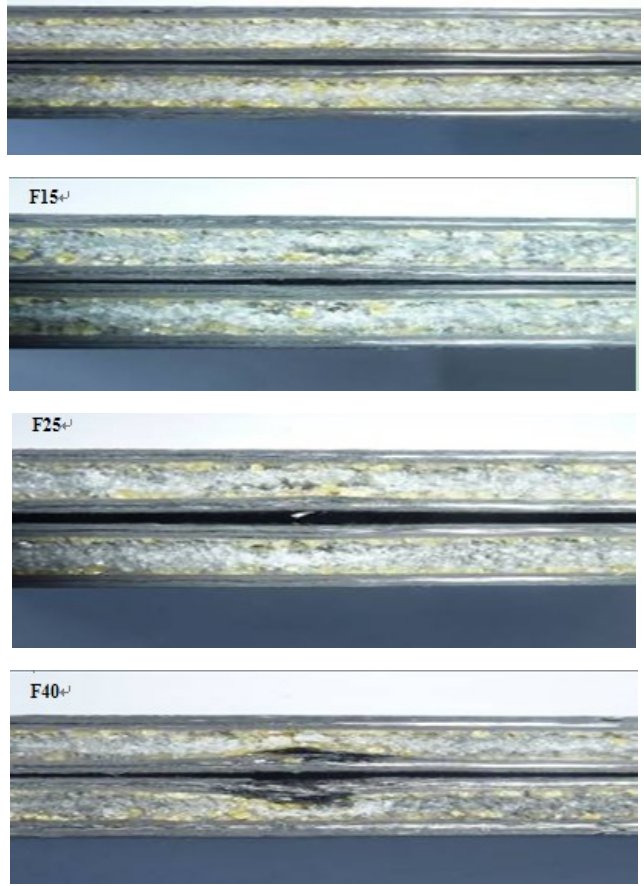

(b) Quasi-static indentation testing (the maximum force of impact testing)

Fig. 7. Cross sectional views of sandwiches subjected to low-velocity impact and quasi-static indentation testing. 


\section{CONCLUSION}

Following conclusions were drawn from this work:

1) Compared with damage area, dent depth was suitable as damage parameter to demonstrate the damage equivalence of foam core sandwich composites subjected to low-velocity impact and quasi-static indentation testing. The length of a submitted paper should be commensurate with the importance, or appropriate to the complexity, of the work. For example, an obvious extension of previously published work might not be appropriate for publication or might be adequately treated in just a few pages.

2) The dent depth of sandwich composites after impact occurred knee point at $0.30 \mathrm{~mm}$, while after quasi-static indentation at $0.27 \mathrm{~mm}$. The knee points of the two kinds of tests were very close and the variable tendency of the dent depth of the two tests was similar, which indicated that quasi-static indentation test could replace low-velocity impact test. During the stage of low impact energy (less than $12 \mathrm{~J}$ ), quasi-static indentation force was seen as approximately equal to the maximum force caused by impact process of corresponding impact energy.

3) An analysis of damage process curves of the two kinds of tests showed the variable tendency of the load was so similar that low-velocity impact test and quasi-static indentation test were equivalent.

4) Damage behavior of the two kinds of tests looked similar seen from cross sectional views of damaged samples. Initially collapse of foam appeared, and then crack of foam occurred, finally fiber breakage and delamination appeared on the upper facing.

\section{REFERENCES}

[1] J. R. Vinson, "Sandwich structures," Appl. Mech. Rev.vol.54, no.3, pp. 201-12, 2001.

[2] A. Shipsha, "Failure mechanisms and modeling of impact damage in sandwich beams-a 2D approach: Part I-experimental investigation," $J$ Sandwich Struct Mater, vol. 5, no. 1, pp. 7-32, 2003.

[3] D. Zenkert, "An introduction to sandwich construction," London: Chameleon Press Ltd, 1995.

[4] S. Abrate, "Impact on composite structures," Cambridge: Cambridge University Press, 1998.
[5] W. C. Jackson and C. C. Poe, "The Use of Impact Force as a Scale Parameter for the Impact Response of Composite Laminates," NASA Technical Memorandum 104189, January, 1992.

[6] Y. S. Kwon and B. V. Sankar, "Indentation-Flexure and Low-Velocity Impact Damage in Graphite Epoxy Laminates," J. Compos. Techol. Res., vol. 15, no. 2, pp. 101-111, 1993.

[7] H. Kaczmarek and S. Maison, "Comparative Ultrasonic Analysis of Damage in CFRP Under Static Indentation and Low-Velocity Impact," Compos. Sci. Technol., vol. 51, pp. 11-26, 1994.

[8] S. M. Lee and P. Zahuta, "Instrumented Impact and Static Indentation of Composites,” J. Compos. Mater., vol. 25, pp. 204-222, 1991.

[9] P. O. Sjoblom, T. J. Hartness, and T. M. Cordell, "On Low-Velocity Impact Testing of Composite Materials," J. Compos. Mater., vol. 22, pp. 30-52, 1998.

[10] A. T. Nettles, M. J. Douglas, "A Comparison of Quasi-Static Indentation Testing to Low Velocity Impact Testing," Composite Materials: Testing, Design, and Acceptance Criteria, ASTM STP 141, 2002.

[11] P. A. Lagace, J. E. Williamson, and P. H. Wilson, "Wolf, E. and Thomas, S., A Preliminary Proposition for a Test Method to Measure (Impact) Damage Resistance," J. reinf. Plast. Compos., vol. 12, pp. 584-601, 1993.

[12] W. Elber, "Failure Mechanics in Low-Velocity Impacts on Thin Composite Plates," NASA Technical Paper, 2152, 1983.

[13] A. L. Highsmith, "A Study of the Use of Contact Loading to Simulate Low Velocity Impact," NASA Contractor Report, 97-206121, 1997.

[14] R. Ferri and B. V. Sankar, "Static indentation and low velocity impact tests on sandwich plates," Proc ASME Aerospace Div, vol. 55, pp. 485-490, 1997.

[15] C. J. Lindholm and O. T. Thomsen, "sandwich structures 7," Advancing with sandwich Structures and Materials, Netherlands, pp. 635-642, 2005.

[16] ASTM D 7136-05, Test method for measuring the damage resistance of a fiber-reinforced polymer-matrix composite to a drop-weight impact event $[\mathrm{S}]$

[17] ASTM D 6264-98(04), Test method for measuring the damage resistance of a fiber-reinforced polymer-matrix composite to a concentrated quasi-static indentation force $[\mathrm{S}]$

[18] X. Yang, Z. Shen, and Y. Yan, "New methodology for evaluating toughness of composite laminates-investigation of damage resistance[J]," Chinese Journal of Aeronautics, vol. 16, no. 2, pp: 73-79, 2003. (in Chinese)

[19] Z. Shen, Y. Chai, S. C. Yang, et al, 'The outline of new specifications on strength of composite air-craft structures[J]," Acta Aeronaut. et Astronaut. Sin., vol. 27, no. 5, pp: 784-788, 2006. (in Chinese)

[20] Z. Shen, S. C. Yang, P. H. Chen, "Behaviors of composite materials to withstand impact and structural compressive design allowableness[J]," Acta Aeronaut. et Astronaut. Sin., vol. 28, no. 3, pp: 561-566, 2003. (in Chinese)

[21] Z. Shen, L. Ye, J. Wang, et al, "Characterization of damage resistance and damage tolerance behaviour of composite laminates[J]," Acta Mater. Compos. Sin., vol. 21, no. 5, pp. 140-145, 2004. (in Chinese) 